\title{
Caracterización fisico-quimica y actividad antimicrobiana de la secreción mucosa de Achatina fulica
}

\author{
Physicochemical characterization and antimicrobial \\ activity of mucus of Achatina fulica
}

Andrés E Pereira $\mathrm{P}^{1}$, Arley Rey $\mathrm{P}^{1}$, Jenny P López $\mathrm{R}^{1}$, Jennifer P Castro $\mathrm{O}^{1}$, Nelson Uribe $\mathrm{D}^{1}$

Forma de citar: Pereira AE, Rey A, López JP, Castro JP, Uribe N. Caracterización físico-química y actividad antimicrobiana de la secreción mucosa de Achatina fulica. Rev Univ Ind Santander Salud. 2016; 48(2): 188-195. DOI: http://dx.doi.org/10.18273/revsal.v48n2-2016003 @(c) (1) Ð

\section{RESUMEN}

Introducción: El caracol gigante africano Achatina fulica es reconocido como una plaga que afecta la biodiversidad, la Salud Pública y la productividad. Sin embargo, se ha demostrado que su secreción mucosa tiene propiedades cosméticas, cicatrizantes y antimicrobianas. Objetivo: Determinar las características físico-químicas y evaluar la actividad antimicrobiana de la secreción mucosa de Achatina fulica. Metodología: Se hicieron pruebas bioquímicas para la determinación cualitativa y cuantitativa de glúcidos, proteínas y lípidos. Se determinó el contenido de Calcio, Potasio, cloruros, Sodio y Magnesio. Se midieron los parámetros de conductividad eléctrica, potencial óxido-reducción, saturación de oxígeno, oxígeno total disuelto, $\mathrm{pH}$, sólidos disueltos totales. Se realizó un ensayo de actividad antibacteriana por la técnica de microdilución en caldo. Resultados: Se encontraron glúcidos en concentraciones de 582 $\mu \mathrm{g} / \mathrm{mL}$ en la fracción soluble y de $62.1 \mu \mathrm{g} / \mathrm{mL}$ en la fracción de mucinas, y proteínas en concentraciones de $836 \mu \mathrm{g} / \mathrm{mL}$ en la fracción soluble y de $1413 \mu \mathrm{g} / \mathrm{mL}$ en la fracción de mucinas. Se observó actividad antimicrobiana frente a las tres cepas ensayadas. Streptococcus agalactiae alcanzó un MIC90 a una concentración de 3,6 mg/mL con la fracción de mucinas; Staphylococcus aureus resistente a meticilina tuvo un MIC50 de 3,3 mg/mL y Escherichia coli un MIC 70 de $3.8 \mathrm{mg} / \mathrm{mL}$. Conclusiones: Se reportan por primera vez las características físicas y los oligoelementos presente en la secreción de Achatina fulica. La actividad antibacteriana obtenida frente a cepas Gram positivas y Gram negativas plantea la necesidad de realizar estudios para purificar las moléculas con dicha actividad, conocer los mecanismos de acción y establecer la inocuidad, entre otros.

Palabras clave: Achatina fulica, secreción mucosa, Actividad antimicrobiana, Caracterización fisicoquímica.

\footnotetext{
ABSTRACT

Introduction: The giant African snail Achatina fulica is recognized as a scourge affecting biodiversity, public health and productivity. However, it has been shown that the mucus has cosmetic, healing and

1. Universidad Industrial de Santander. Bucaramanga, Colombia

Correspondencia: Nelson Uribe D. Dirección: Escuela de Microbiología. Facultad de Salud - UIS, Edificio Roberto Serpa.

Correo electrónico: nelurdel@uis.edu.co. Teléfono: +57 6344000 Ext. 3417 
antimicrobial properties. Objective: Determine physico-chemical characteristics and evaluate the antimicrobial activity of the mucus. Methodology: Qualitative and quantitative determinations of carbohydrates, proteins and lipids were made by biochemical tests. Using multiparameter meter parameters of electrical conductivity, redox potential, oxygen saturation, the total dissolved oxygen, $\mathrm{pH}$, total dissolved solids were measured. Content of Calcium, Potassium, chloride, Sodium and Magnesium was determined. Antibacterial activity assay was performed by broth microdilution method against Gram positive and Gram negative bacteria. Results: Carbohydrates were found in concentrations of $582 \mu \mathrm{g} / \mathrm{mL}$ in soluble fraction and $62.1 \mu \mathrm{g} / \mathrm{mL}$ in mucin fraction, and protein concentration of $836 \mu \mathrm{g} / \mathrm{mL}$ in the soluble fraction and $1413 \mu \mathrm{g} / \mathrm{mL}$ in mucin fraction. Antimicrobial activity was demonstrated against the three strains tested. Streptococcus agalactiae reached MIC90 at a concentration of $3.6 \mu \mathrm{g} / \mathrm{mL}$ with mucin fraction; Methicillin-Resistant Staphylococcus aureus had a MIC50 of $3.3 \mathrm{mg} / \mathrm{mL}$ and Escherichia coli had a MIC70 of $3.8 \mathrm{mg} / \mathrm{mL}$. Conclusions: This is the first report of the physical and trace elements in the secretion of Achatina fulica. The antibacterial activity obtained against Gram positive and Gram negative strains raises the need for studies to purify the molecules with such activity, understanding the mechanisms of action and establish the safety, among others.

Keywords: Achatina fulica, mucus, antimicrobial activity, physico-chemical characterization

\section{INTRODUCCIÓN}

Los Mollusca son un amplio phylum de animales invertebrados, dentro de los cuales se encuentran los caracoles y las babosas. Los caracoles son gasterópodos de cuerpo blando constituido por una cabeza y un pie aplanado.

Achatina fulica, conocido como el caracol gigante africano es una especie terrestre nativa de África oriental y nororiental, reportado en Asia, Oceanía y América ${ }^{1}$ e incluida dentro de las 100 especies exóticas invasoras más dañinas del mundo por la Unión Internacional para la Conservación de la Naturaleza (UICN) ${ }^{2}$.

Este molusco es una amenaza con impactos directos e indirectos sobre los ecosistemas, la economía y la salud humana. Es considerado como una de las peores plagas agrícolas a nivel mundial ${ }^{3}$. Produce desequilibrio en los ecosistemas donde es introducido, su voracidad, altas tasas de reproducción y elevada capacidad de adaptación a diferentes condiciones ambientales le brindan ventajas competitivas frente a otras especies de caracoles nativas representando un gran riesgo para la biodiversidad. Desde el punto de vista de la Salud Pública, adquiere importancia al ser transmisor de diversos patógenos para el ser humano ${ }^{4}$.

A. fulica repta sobre una variedad de superficies altamente contaminadas siendo el pie el principal sitio de entrada de parásitos y bacterias $^{5}$. Pese a que carece de inmunidad adaptativa, ha desarrollado un sistema de protección contra un amplio rango de patógenos. Estos mecanismos incluyen desde las barreras físicas hasta el sistema inmune innato. El cuerpo de los caracoles está cubierto por una película rica en sustancias mucosas, las cuales están involucradas en mantener la humedad y prevenir la evaporación, así como ayudar a disminuir la fricción del movimiento y proteger contra las injurias mecánicas ${ }^{6}$.

Adicionalmente algunas funciones bioquímicas, no completamente esclarecidas, pueden estar involucradas, como la activación de fibroblastos por medio de la producción de glicoxil-diurea, la producción de antiproteasas, y la secreción de defensinas capaces de actuar contra una gran variedad de agentes infecciosos ${ }^{7,8}$.

La secreción mucosa de los gasterópodos y dentro de ellos $A$. fulica, es una sustancia viscosa compuesta principalmente por proteínas altamente glicosiladas denominadas mucinas, que además contiene Calcio, Potasio, Cloruros, Sodio, Magnesio y Fósforo, componentes necesarios para la mineralización y regeneración de la concha?.

Otros componentes del moco son los polisacáridos formados por unidades repetidas como en los glucosaminoglucanos. Tales polisacáridos son referidos como mucopolisacaridos que son el mayor constituyente de estas secreciones y del cual se han propuesto un número importante de funciones biológicas como: la inhibición de la angiogénesis en los procesos inflamatorios por medio de acharan sulfato ${ }^{10}$; la inhibición del crecimiento microbiano debido a la presencia de péptidos y lectinas involucradas en la defensa inmunológica de estos moluscos $^{11,12,13}$. Existen otros componentes como la alantoína que tiene propiedades estimulantes de la epitelización de la piel por estímulo de la proliferación de fibroblastos ${ }^{14}$, el ácido glucónico que actúa como exfoliante natural de la piel, el colágeno y la elastina que confieren tersura a la piel; y enzimas fibrinolíticas que limpian los microcapilares favoreciendo la oxigenación 
y nutrición de los tejidos, y con ello un remodelamiento vascular adecuado ${ }^{15,16,17}$.

Aunque no se han hecho estudios detallados sobre las características físicas y bioquímicas de la secreción mucosa sí se han identificado Péptidos Antimicrobianos (PAMs) que pueden constituir una alternativa al problema de la resistencia que presentan muchos microorganismos ${ }^{13,18}$.

El estudio de la secreción mucosa es la mejor manera de convertir un problema en oportunidades para el avance científico con miras a obtener productos que solucionen necesidades de importancia biomédica, teniendo en cuenta sus propiedades cosméticas, cicatrizantes y antimicrobianas demostradas. Para esto es necesario caracterizar la secreción, determinando sus propiedades físicas y su composición bioquímica.

Este trabajo reporta la caracterización físico-química de la secreción mucosa y la evaluación de la actividad antimicrobiana de las fracciones soluble y mucoproteica.

\section{METODOLOGÍA}

\section{Selección de los caracoles y obtención de la secreción mucosa}

Para la recolección de la secreción mucosa se ubicó un foco de Achatina fulica en el "Parque Ecológico La Flora" del municipio de Bucaramanga, y en jardines de zonas residenciales del municipio de Floridablanca, Santander, Colombia. Los caracoles se identificaron como miembros de esta especie por las características morfológicas de la concha. Se seleccionaron aquellos que medían entre 5 y $12 \mathrm{~cm}$ de longitud. La secreción mucosa se obtuvo por estimulación directa del pie del caracol con corriente eléctrica de $9 \mathrm{~V}$ a intervalos de 3060 segundos y se recogió en recipientes estériles, previa limpieza del pie con solución yodada. De cada caracol se obtuvo entre 3 y $6 \mathrm{~mL}$ de secreción mucosa, la cual se recolectó formando una sola colección.

\section{Fraccionamiento del moco}

Las fracciones soluble y mucoproteica se obtuvieron de acuerdo al procedimiento de Iguchi et al, 1981 ${ }^{11}$. La fracción soluble en agua (FS) fue obtenida por la adición de dos partes de agua a una parte de secreción mucosa y su posterior agitación durante 24 horas. Una vez solubilizada completamente la secreción en agua se centrifugó a $8000 \mathrm{~g}$ por 30 minutos. El sobrenadante fue referenciado como FS. La fracción mucoproteica (FM) fue obtenida adicionando tres partes de etanol, con pureza de $99.9 \%$, como precipitante de las mucoproteínas (mucinas) a una parte de la fracción soluble (FS); esta mezcla se centrifugó a 2500 g por 30 minutos y el precipitado obtenido fue referenciado como FM.

\section{Determinación de las propiedades físicas}

Se midieron los parámetros de conductividad, sólidos totales disueltos (STD), oxígeno disuelto, saturación de oxígeno y potencial de óxido-reducción (ORP) utilizando el medidor multiparamétrico HMP6 de $H A C H^{\circledR}$. El pH de la secreción mucosa y la fracción soluble se determinó con pHmetro (Schott lab 850). Además se evaluó la densidad de la secreción mucosa y de la fracción soluble por picnometría. Como propiedad intensiva se determinó la solubilidad de la secreción y de las dos fracciones en agua, cloroformo y éter, para lo cual se utilizaron dos volúmenes secreción por cada volumen de solvente.

\section{Determinación cualitativa de carbohidratos, proteínas y lípidos}

Se usaron pruebas bioquímicas para la valoración cualitativa de carbohidratos (Lugol, Benedict, Antrona, DNS, Ropes), proteínas (Biuret) y lípidos (saponificación, Hübl, Lieberman, Sudan III) en la secreción mucosa, la fracción soluble (FS) y la fracción mucoproteica (FM). Para cada ensayo se hicieron controles positivos y negativos siguiendo los lineamientos básicos para cada una de las pruebas según las guías de la Facultad de Ciencias Biológicas de la Universidad Autónoma de Nuevo León ${ }^{19}$.

\section{Cuantificación de proteínas y carbohidratos}

Se cuantificaron proteínas y carbohidratos en la fracción soluble y mucoproteica. La concentración de proteínas se calculó mediante el método de Bradford, usando albúmina de suero bovino para la construcción de la curva patrón. La cuantificación de carbohidratos se realizó por el método de Fenol Ácido Sulfúrico usando soluciones de glucosa, almidón, lactosa y sacarosa como glúcidos patrón ${ }^{20}$.

\section{Composición inorgánica del moco}

Los componentes inorgánicos presentes en la secreción mucosa y en la fracción soluble se cuantificaron en el Laboratorio Químico de Consultas Industriales (LQCI) de la Universidad Industrial de Santander utilizando técnicas de absorción atómica. Se determinó la concentración de Calcio, Potasio, Sodio, Hierro, Magnesio, Fósforo y Cloruros por ser los oligoelementos 
más representativos en las muestras biológicas. Para cada una de las determinaciones se siguió el manual de procedimientos del (LQCI) según la Normas Técnicas Colombiana (NTC), Método Estándar (SM), y la Agencia de Protección Ambiental de los Estados Unidos (EPA).

\section{Ensayo de actividad antimicrobiana}

Se evaluó la cinética de crecimiento de Escherichia coli, Staphylococcus aureus resistente a meticilina (SARM) y Streptococcus agalactiae ATCC 12403 en presencia $\mathrm{y}$ ausencia de la fracción soluble y de diferentes concentraciones de la fracción mucoproteica $(3,8 \mathrm{mg} /$ $\mathrm{mL} ; 1,9 \mathrm{mg} / \mathrm{mL} ; 0,95 \mathrm{mg} / \mathrm{mL} ; 0,475 \mathrm{mg} / \mathrm{mL} ; 0,2378 \mathrm{mg} /$ $\mathrm{mL})$. Se determinó la Concentración Mínima Inhibitoria empleando el método de microdilución en caldo con placas microtituladoras de acuerdo al procedimiento establecido en el estándar CLSI-M07-A9-2012 ${ }^{21}$.

Se repicaron las cepas en agar nutritivo 24 horas antes de hacer el ensayo y se prepararon preinóculos de las cepas seleccionadas haciendo repiques en caldo infusión cerebro corazón (BD Biosciences) los cuales fueron llevados a agitación constante a $200 \mathrm{rpm}$ e incubación a $37^{\circ} \mathrm{C}$ durante 12 horas. Antes del ensayo las fracciones se filtraron al vacío utilizando filtros con poros de $0.45 \mu \mathrm{m}$ y los preinóculos se ajustaron a una absorbancia entre 0.08 y 0.1 a $595 \mathrm{~nm}$. La fracción mucoproteica se rotoevaporó a $50 \mathrm{~atm}$ y $100 \mathrm{rpm}$ a $28^{\circ} \mathrm{C}$ para preparar soluciones con diferente concentración de proteínas. El ensayo incluyó: medio de cultivo sin las fracciones como control negativo, blanco del medio con las fracciones, y las pruebas del microorganismo con cada una de las fracciones. Cada ensayo se hizo por triplicado.

\section{RESULTADOS}

\section{Características físico-químicas}

Tabla 1. Propiedades físico-químicas de la Fracción Soluble

\begin{tabular}{lcc}
\hline Propiedad física & Valor & Unidades \\
\hline pH & 6,98 & \\
Conductividad & 3368 & $\mu \mathrm{S}$ \\
Sólidos Disueltos Totales (TDS) & 2571 & $\mathrm{ppm}$ \\
Oxígeno Disuelto & 4,56 & $\mathrm{ppm}$ \\
\% saturación del oxígeno Disuelto & 43,9 & $\%$ \\
Potencial de óxido-Reducción (ORP) & 102 & $\mathrm{mV}$ \\
Densidad & 0.99 & $\mathrm{~g} / \mathrm{mL}$ \\
\hline
\end{tabular}

Tanto la secreción mucosa como la fracción mucoproteica fueron solubles en agua e insolubles en cloroformo y éter.

\section{Determinación cualitativa de biomoléculas}

Las pruebas de Lugol, Benedict y DNS fueron negativas indicando la ausencia de almidones y azúcares reductores. Se evidenció la presencia de mucoproteínas (glucoproteínas) en la fracción soluble y mucoproteica mediante la prueba de Ropes, la cual refleja el grado de polimerización de las mucinas. La prueba de Antrona fue positiva revelando la presencia de ácidos urónicos, pentosas y/o hexosas en las fracciones soluble y mucoproteica.

Las pruebas de saponificación, Hübl, Lieberman y Sudan III fueron negativas, descartando la presencia de ácidos grasos saponificables, ácidos grasos insaturados, derivados del ciclopentanoperhidrofenantreno y triglicéridos, respectivamente.

La prueba de Biuret para la determinación de proteínas en la secreción mucosa y sus fracciones fue positiva con concentraciones entre 0,5 y $2 \mathrm{mg} / \mathrm{mL}$ de acuerdo a los patrones de albúmina bovina.

\section{Cuantificación de carbohidratos y proteínas}

Se encontraron carbohidratos en concentraciones de $582 \mu \mathrm{g} / \mathrm{mL}$ en la fracción soluble y de $62.1 \mu \mathrm{g} / \mathrm{mL}$ en la fracción mucoproteica, y proteínas en concentraciones de $836 \mu \mathrm{g} / \mathrm{mL}$ en la fracción soluble y de $1413 \mu \mathrm{g} / \mathrm{mL}$ en la fracción mucoproteica.

\section{Determinación de moléculas inorgánicas}

Tabla 2. Determinación de moléculas inorgánicas

\begin{tabular}{lcc}
\hline Oligoelementos & $\begin{array}{c}\text { Resultado } \\
(\mathbf{m g} / \mathrm{L})\end{array}$ & Método \\
\hline Calcio & 1257,71 & $\begin{array}{c}\text { Absorción Atómica/SM 3030 } \\
\text { E y SM 3111 D }\end{array}$ \\
Fósforo & 3,13 & $\begin{array}{c}\text { Espectrofotométrico/SM 4500- } \\
\text { P C }\end{array}$ \\
Potasio & 528,76 & $\begin{array}{c}\text { Absorción Atómica/SM 3030 } \\
\text { E y SM 3111 B }\end{array}$ \\
Sodio & 139,40 & $\begin{array}{c}\text { Absorción Atómica/SM 3030 } \\
\text { E y SM 3111 B }\end{array}$ \\
Cloruros & 502,46 & $\begin{array}{c}\text { Argentométrico/ SM 4500- } \\
\text { Cl- B }\end{array}$ \\
Hierro & $<$ L.D & $\begin{array}{c}\text { Absorción Atómica/SM 3030 } \\
\text { E y SM 3111 B }\end{array}$ \\
Magnesio & 68,12 & $\begin{array}{c}\text { Absorción Atómica/SM 3030 } \\
\text { E y SM 3111B }\end{array}$ \\
\hline
\end{tabular}

\section{Ensayo de actividad antimicrobiana}

Tanto la FS como la FM presentaron actividad antimicrobiana contra las bacterias estudiadas. El mayor porcentaje de inhibición se obtuvo en 
Caracterización físico-química y actividad antimicrobiana de la secreción mucosa de Achatina fulica

Streptococcus agalactiae ATCC 12403 con una Concentración Mínima Inhibitoria $90\left(\mathrm{CMI}_{90}\right)$ de 3,6 $\mathrm{mg} / \mathrm{mL}$ de proteínas en la fracción mucoproteica. Tabla 3. El efecto inhibitorio es directamente proporcional a la concentración de la fracción mucoproteica. La fracción soluble presenta un comportamiento de inhibición similar a la fracción de mucinas entre las concentraciones de $0,95 \mathrm{mg} / \mathrm{mL}$ y $1,9 \mathrm{mg} / \mathrm{mL}$. Figura 1 .
Tabla 3. Concentración Mínima Inhibitoria (CMI) de la fracción mucoproteica frente a las cepas evaluadas por el método de microdilución en caldo ${ }^{21}$.

\begin{tabular}{lccc}
\hline Microorganismo & $\begin{array}{c}\text { CMI 50 } \\
(\mathbf{m g} / \mathbf{m L})\end{array}$ & $\begin{array}{c}\text { CMI 70 } \\
(\mathbf{m g} / \mathbf{m L})\end{array}$ & $\begin{array}{c}\text { CMI 90 } \\
(\mathbf{m g} / \mathbf{m L} \mathbf{)}\end{array}$ \\
\hline $\begin{array}{l}\text { Streptococcus agalactiae } \\
\begin{array}{l}\text { Staphylococcus aureus } \\
\text { resistente a meticilina }\end{array}\end{array}$ & 2,1 & - & 3,6 \\
\begin{tabular}{l} 
Escherichia coli \\
\hline
\end{tabular} & 2,4 & - & - \\
\hline
\end{tabular}
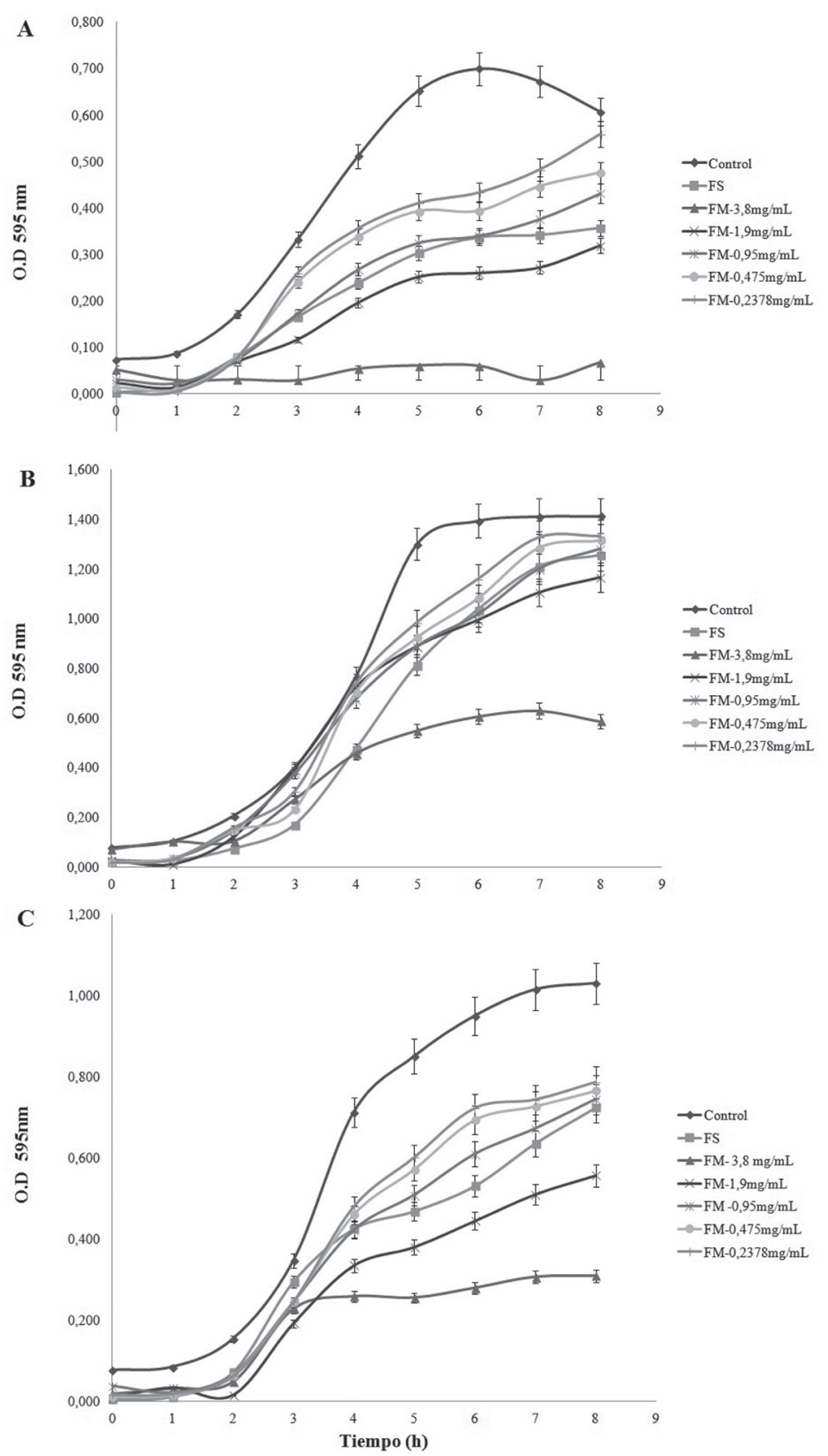

Figura 1. Ensayo de actividad antimicrobiana. A. Efecto antibacteriano de las fracciones frente a Streptococcus agalactiae ATCC 12403. B. Efecto antibacteriano de las fracciones frente a Staphyloococcus aureus resistente a meticilina (SARM). C. Efecto antibacteriano de las fracciones frente a Escherichia coli. 


\section{DISCUSIÓN}

Estudiar propiedades físicas, químicas y biológicas de la secreción mucosa de Achatina fulica reviste gran importancia pues aporta argumentos para evaluar usos alternativos y riesgos para la salud humana. En la literatura revisada no encontramos publicaciones que reporten un estudio de las características físicas de la secreción mucosa de $A$. fulica. Nuestros hallazgos de $\mathrm{pH}$ neutro y alta cantidad de oxígeno (reflejada en el total de oxígeno disuelto y en el porcentaje de saturación de oxígeno) convierten la secreción en un medio con alto potencial de óxido-reducción favorable a reacciones de transferencia de electrones, que pueden ser utilizadas para la obtención de energía por un amplio grupo de microorganismos. Esto es relevante para la salud pública, por el riesgo de transmisión de patógenos a través de la secreción, pues se conoce que el molusco es omnívoro exponiéndolo a un amplio rango de microorganismos al desplazarse por diferentes superficies en entornos urbanos, suburbanos y silvestres ${ }^{22}$.

La FS fue positiva para proteínas y carbohidratos, lo cual, guarda relación con la positividad de la prueba de Ropes sugiriendo la presencia de complejos glucoproteicos pertenecientes a la familia de las mucinas con un alto contenidos de glucosaminoglicanos del tipo urónicos, como lo demuestra la prueba de Antrona. La totalidad de los carbohidratos presentes en la secreción mucosa se encuentran formando compuestos con proteínas ya sea en forma de mucoproteínas o ácidos urónicos, como lo demuestran las pruebas de Ropes, Antrona y Biuret. La concentración de carbohidratos fue mayor en la FS que en la mucoproteica, lo cual está relacionado con el alto contenido de ácidos urónicos que son altamente solubles. En la fracción mucoproteica no fueron detectados significativamente ya que estos glucosaminoglicanos se encuentran fuertemente unidos a las proteínas dificultando su detección ${ }^{19}$.

Los resultados de la cuantificación de carbohidratos y proteínas, al igual que los resultados de otros estudios, muestran que la diferencia en la proporción de carbohidratos y proteínas en la FS refleja la variación en la longitud de la cadena de carbohidratos ${ }^{11}$.

No encontramos en la literatura revisada estudios de moléculas inorgánicas en las secreciones mucosas de los caracoles; sin embargo, se conoce que éstas son importantes por ser oligoelementos que participan en la mineralización y regeneración de la concha y el pie, siendo el calcio el principal constituyente de la concha9. La concentración media de proteínas cuantificada por el método de Bradford fue de $2 \mathrm{mg} / \mathrm{mL}$, menor a la encontrada en otros estudios que reportan concentraciones máximas de $4 \mathrm{mg} / \mathrm{mL}$ por el método de micro-Biuret ${ }^{11}$. Este resultado es significativo ya que el método de Bradford presenta limitaciones a la hora de cuantificar la masa de proteínas totales; por ende se hace necesario usar métodos analíticos más específicos y sensibles.

La cinética de crecimiento reveló una inhibición en la fase de latencia de la curva de crecimiento de $S$. agalactiae y E. coli aproximadamente en las dos primeras horas; sin embargo, esto no sucede para SARM. En la fase exponencial se evidenció una disminución de la inhibición para las tres cepas bacterianas, especialmente para $S$. agalactiae, donde se obtuvo un mayor porcentaje de inhibición en la fracción de mucinas de $3,8 \mathrm{mg} / \mathrm{mL}$ de proteínas.

La capacidad inhibidora se encuentra en las mucoproteínas de la secreción mucosa, ya que cuando se precipitan y se extraen en la fracción mucoproteica la actividad antimicrobiana se incrementa. Esto se evidencia al comparar con los resultados de otros estudios donde la fracción mucoproteica presenta mayor efecto inhibitorio que la fracción soluble ${ }^{11}$. Debido a la naturaleza gluco-proteica de la FM, podría pensarse que los mecanismos de inhibición del crecimiento bacteriano guardan relación con el grupo de antibiótico glucosaminoglicanos, los cuales tienen actividad contra bacterias Gram negativas y actúan sinérgicamente contra bacterias Gram positivas ${ }^{23}$. Nuestros resultados se relacionan con los encontrados por Iguchi et al. donde la FS y FM mostraron actividad antibacteriana tanto para las bacterias Gram positivas Bacillus subtilis y Staphylococcus aureus, como para las bacterias Gram negativas, Escherichia coli y Pseudomonas aeruginos $a^{11}$.

Este trabajo hace un aporte significativo al estudio de la secreción mucosa de Achatina fulica ya que no se han reportado con detalle las características físicas, así como la presencia de moléculas inorgánicas.

El estudio in vitro de la actividad antimicrobiana reveló inhibición del crecimiento de bacterias Gram positivas y Gram negativas de interés en clínica humana. Estos resultados pueden ser tomados como insumo para posteriores estudios que incluyan: purificar los péptidos con actividad antimicrobiana, esclarecer los mecanismos de acción, obtener péptidos recombinantes y determinar efectos citotóxicos y hemolíticos, entre otros, buscando contribuir a la problemática de la resistencia a los antimicrobianos. 


\section{AGRADECIMIENTOS}

COLCIENCIAS, Convocatoria 617 de 2013. Jóvenes investigadores e innovadores y Semilleros de investigación. Con apoyo de las Escuelas de Microbiología, Química y Biología de la Universidad Industrial de Santander.

\section{CONSIDERACIONES ÉTICAS}

El proyecto contó con el aval del Comité de Ética para la Investigación Científica (CEINCI) de la Universidad Industrial de Santander.

\section{CONFLICTOS DE INTERÉS}

Los autores no manifiestan ningún conflicto de interés.

\section{REFERENCIAS}

1. Carvalho de Vasconcelos M, Pile E. Ocorrência de Achatina fulica no Vale do Paraíba, Estado do Rio de Janeiro, Brazil. Rev Saúde Pública. 2001; 35(6): 582-584.

2. Courchamp F. Alien species: monster fern makes IUCN invader list. Nature. 2013; 498(7452): 37. DOI: $10.1038 / 498037 \mathrm{a}$.

3. Raut S, Barker G. Achatina fulica Bowdich and other Achatinidae as pests in tropical agriculture. In: Barker GM, 2002 Molluscs as crop pests. CABI Publishing, Wallingford, $\mathrm{p} 55114$.

4. Lima AR, Mesquita SD, Santos SS, Aquino ER, Rosa Lda R, Duarte FS, et al. Alicata disease: neuroinfestation by Angiostrongylus cantonensis in Recife, Pernambuco, Brazil. Arq Neuropsiquiatr. 2009; 67(4): 1093-1096.

5. Hancock R, Brown K, Mookherjee N. Host defence peptides from invertebrates emerging antimicrobial strategies. Immunobiology. 2006; 211(4): 315-322.

6. Tyrakowski T, Kaczorowski P, Pawłowicz W, Ziółkowski M, Smuszkiewicz P, Trojanowska I, et al. Discrete movements of foot epithelium during adhesive locomotion of a land snail. Folia Biol (Krakov). 2012; 60(1-2): 99-106.

7. Biswas $C$, Sinha D, Madal C. Investigation on interaction of Achatinin, a 9-O-acetyl sialic acidbinding lectin, with lipopolysaccharide in the innate immunity of Achatina fulica snails. Mol Immunol. 2000; 37(12-13): 745-754.

8. Guerrero M, Miranda Vargas F, Ramírez Rosero A, Nuque ML, Salazar Bolaños E, Tomalá Briones M, et al. Meningitis eosinofilíca por Angiostrongylus cantonensis. Reporte de caso de autopsia. Rev
Medicina. 2008; 13(4): 313-318.

9. Schreurs J, Investigations on the biology, ecology and control of the Giant African Snail in West New Guinea. 1963, Unpublished report, Manokwari Agricultural Research Station, $18 \mathrm{p}$.

10. Ghosh A K, Hirasawa N, Lee YS, Kim YS, Shin $\mathrm{KH}$, Ryu N, et al. Inhibition by acharan sulphate of angiogenesis in experimental inflammation models. Br J Pharmacol. 2002; 137(4): 441-448. DOI: 10.1038/sj.bjp.0704886.

11. Iguchi SM, Aikawa, T, Matsumoto JJ. Antibacterial activity of snail mucus mucin. Comp Biochem Physiol. 1982; 72(3): 571-574.

12. Kubota Y, Watanabe Y, Otsuka H, Tamiya T, Tsuchiya T, Matsumoto JJ. Purification and characterization of an antibacterial factor from snail mucus. Comp Biochem Physiol C. 1985; 82(2): 345-348.

13. Zhong J, Wang W, Yang X, Yan X, Liu R. A novel cysteine-rich antimicrobial peptide from the mucus of the snail of Achatina fulica. Peptides. 2013; 39: 1-5. DOI: 10.1016/j.peptides.2012.09.001.

14. Lira Cortes AJ. Determinación de alantoína en secreción de caracol chileno (Helix aspersa müller). variaciones en su concentración según tipo de alimentación y época de obtención en el año. 2008. Universidad Austral de Chile.

15. Martins MF, Macedo Caetano FA, Sírio OJ, Mizusaki Yiomasa M, Ioshie Mizusaki C, Deadame de Figueiredo L, et al. Avaliação do reparo de lesões de pele de coelhos tratadas com secreção mucoglicoproteica do escargot Achatina. Braz J Vet Res Anim Sci. 2003; 1(1):1-11. DOI: org/10.1590/ S1413-95962003000900009.

16. Sírio, O. Verificação da potencialização do efeito cicatrizante do muco de caracóis do gênero Achatina promovida por dieta a base de Confrei (Symphytum officinale L.). Dissertação, Mestrado. Programa de Pós-Graduação em Nutrição e Produção Animal. Faculdade de Medicina Veterinária. Pirassununga, Universidade de São Paulo, 2005. p.87.

17. Alcântara Santana W, Moura de Melo C, Cordeiro Cardoso J, Pereira-Filho RN, Silva Rabelo A, Prado Reis F, et al. Assessment of Antimicrobial Activity and Healing Potential of Mucous Secretion of Achatina fulica. Int J Morphol. 2012; 30(2): 365373. DOI: $10.4067 /$ S0717-95022012000200001.

18. Hancock R, Brown K, Mookherjee N. Host defence peptides from invertebrates emerging antimicrobial strategies. Immunobiology. 2006; 211(4): 315-322.

19. Rodríguez J, Manual de pruebas bioquímicas, 4 edición, Monterrey, 1987.

20. Albalasmeh AA, Berhe AA, Ghezzehei TA. A new method for rapid determination of carbohydrate 
and total carbon concentrations using UV spectrophotometry, Carbohydr Polym. 2013; 97(2): 253-261. DOI: 10.1016/j.carbpol.2013.04.072.

21. Clinical and Laboratory Standards Institute. Methods for Dilution Antimicrobial Susceptibility Tests for Bacteria That Grow Aerobically. 2002. Wayne, PA. CLSI M07-A9 Vol. 32 No. 2: 18-19.

22. Liboria M, Morales G, Sierra C, Silva I, Pino LA. Primer hallazgo en Venezuela de huevos de Schistosoma mansoni y de otros helmintos de interés en salud pública, presentes en heces y secreción mucosa del molusco terrestre Achatina fulica (Bowdich, 1822). Zootecnia Trop. 2010; 28(3): 383-394.

23. Champe PC, Harvey RA, Ferrier DR. Biochemistry. Lippincott Williams \& Wilkins. 2004. 